\title{
Die Umsetzung der Kreislaufwirtschaft in österreichischen Unternehmen - Praktiken, Strategien und Auswirkungen auf den Unternehmenserfolg
}

\author{
Josef-Peter Schöggl · Lukas Stumpf · Magdalena Rusch · Rupert J. Baumgartner
}

Angenommen: 15. November 2021 / Online publiziert: 10. Dezember 2021

(C) Der/die Autor(en) 2021

Zusammenfassung Das Konzept der Kreislaufwirtschaft befindet sich seit 2015 als einer der wichtigsten Punkte auf der europäischen Agenda. Infolgedessen wurde auch die Forschung zur Rolle von Unternehmen in der Umstellung auf eine Kreislaufwirtschaft in den letzten Jahren erheblich intensiviert. Bislang wird die wissenschaftliche Literatur jedoch von konzeptionellen Arbeiten dominiert und die verfügbare empirische Forschung besteht hauptsächlich aus Einzel- oder Mehrfachfallstudien. Empirische Studien, die auf größeren Stichproben beruhen, sind selten und bisher hat keine Studie den Stand der Umsetzung der Kreislaufwirtschaft in österreichischen Unternehmen zum Gegenstand gehabt. Diese Forschungslücke wird durch die vorliegende Studie geschlossen, die die Ergebnisse von Telefoninterviews mit 120 GeschäftsführerInnen und 100 Nachhaltigkeitsverantwortlichen österreichischer Unternehmen darlegt. Der Fokus liegt dabei auf Unternehmen des produzierenden Gewerbes. Die Interviewfragen wurden aus der Literatur abgeleitet und bauen auf etablierten Konzepten wie den 10-Rs oder dem ReSOLVE Framework auf. Die Ergebnisse geben erstens Aufschluss über die strategische Kreislaufwirtschaftsorientierung österreichischer Unternehmen, indem sie detailliert aufzeigen, inwieweit Überlegungen zur Kreislauf-

\footnotetext{
J.-P. Schöggl, Bakk.rer.soc.oec., MSc, PhD ( $\varangle) \cdot$ L. Stumpf, BA, MSc $\cdot$ M. Rusch, BA, MSc . Univ.-Prof. Dipl.-Ing. Dr. R. J. Baumgartner Christian Doppler Labor für nachhaltiges Produktmanagement in einer Kreislaufwirtschaft, Institut für Systemwissenschaften, Innovationsund Nachhaltigkeitsforschung, Universität Graz,

Merangasse 18, 8010 Graz, Österreich josef.schoeggl@uni-graz.at
}

wirtschaft in Unternehmensstrategien, Innovationen, Humankapital und Geschäftsführung integriert sind. Zweitens wird der Grad der Umsetzung von 26 spezifischen kreislaufwirtschaftsund nachhaltigkeitsorientierten Praktiken dargestellt. Diese Praktiken decken eine vollständige Lebenszyklusperspektive ab und umfassen Interventionen in verschiedenen Unternehmensbereichen. So wird beispielsweise dargestellt, inwieweit sich Unternehmen für ein nachhaltiges Lieferkettenmanagement engagieren, ihr Produktdesign ändern (z. B. Design für Recycling/ Wiederverwendung/Reparatur, ...), ihre Produktion optimieren (z.B. Verwendung von Rezyklaten, Schließung interner Ressourcenkreisläufe, ...), ihre Geschäftsmodelle ändern (z.B. durch Dematerialisierung oder den Verkauf von Nebenprodukten) oder Post-Consumer-Produkte wiederverwenden bzw. rezyklieren. Drittens wird aufgezeigt, wie CEOs die Auswirkungen der Umsetzung dieser Strategien auf die finanzielle und nachhaltige Gesamtleistung ihrer Unternehmen bewerten. Abschließend wird dargestellt, wie die CEOs die Bereitschaft des österreichischen Marktes für die Kreislaufwirtschaft einschätzen. Dazu wird aufgezeigt, wie die Befragten den Wettbewerbsdruck, die technologischen Entwicklungen und die Nachfrage nach zirkulären Produkten bewerten.

Zusammenfassend zeigt die Studie, dass die Einführung von Kreislaufwirtschaft in österreichischen Unternehmen immer noch in erster Linie auf Compliance und Effizienz ausgerichtet ist. Radikalere Veränderungen auf Produktebene, in der Produktion und vor allem in den Geschäftsmodellen sind selten. Diese und die anderen deskriptiven Erkenntnisse dieser Studie bieten PraktikerInnen und ForscherInnen eine umfassende Annäherung an den Reifegrad der Kreislaufwirtschaft in österreichischen Unternehmen aus ver- schiedenen Perspektiven. Damit kann sie als Grundlage für weitere Forschung und unternehmerisches Engagement bei der Umsetzung der Kreislaufwirtschaft dienen - eine Voraussetzung für die Erschließung der vollen Potenziale einer Kreislaufwirtschaft in Österreich.

Schlüsselwörter Umsetzung der Kreislaufwirtschaft · Empirische Erhebung · Österreich

The implementation of the Circular Economy in Austrian companies - practices, strategies and impacts on corporate success

Abstract The circular economy (CE) concept has been a top priority on the European agenda since 2015. Consequently, research on business transformation towards a CE has grown significantly in the last years. However, up to now, literature is dominated by conceptual research, and available empirical research mainly consists of single or multiple case studies. Empirical studies based on larger samples are rare and, so far, no study has investigated the state of implementation of the circular economy in Austrian firms. This research gap is addressed by this study, which provides the results of telephone interviews with 120 CEOs and 100 sustainability executives of Austrian firms. The focus lies on companies from the manufacturing sector. The interview questions were derived from literature and build on established concepts such as the 10-Rs or the ReSOLVE framework. The results, first, reveal the strategic CE orientation of Austrian companies by detailing to which extent $\mathrm{CE}$ considerations are integrated into corporate strategies, innovation, human resources, and operations. Second, the degree of implementation of 26 specific CE and sustainability-oriented practices is illustrated. These prac- 
tices cover a full life cycle perspective and comprise interventions in different areas of the firm. For instance, it is portrayed to which extent firms engage in sustainable supply chain management, change their product design (e.g., design for recycling/reuse/repair, ...), optimize their production (e.g., use of recyclates, closing of internal resource loops, ...), change their business models (e.g., via dematerialization or the sale of by-products), or reuse/recycle post-consumer products. Third, it is shown how CEOs evaluate the impact of implementing these strategies on the overall financial and sustainability performance of their companies. Finally, the CEOs' perception of the Austrian market's readiness for a $\mathrm{CE}$ is provided. This is done by illustrating how the interviewees evaluated competitive pressure, technological developments, and demand for circular products.

In summary, the study reveals that the CE adoption in Austrian firms is still primarily compliance and efficiency driven. More radical changes on the product level, the production and especially the business models are seldom. These and the other descriptive insights of this study provide practitioners and researchers with a comprehensive approximation of the maturity of the circular economy in Austrian firms from different perspectives. Thus, it may serve as a basis for further research and business engagement on the $\mathrm{CE}$ implementation-a requirement for unlocking the full potentials of a circular economy in Austria.

Keywords Circular economy implementation - Empirical survey · Austria

\section{Einleitung}

Das Konzept der Kreislaufwirtschaft (Circular Economy, CE) nimmt seit 2015 einen zentralen Platz auf der europäischen Agenda ein (European Commission 2015, 2020). Infolgedessen hat die Forschung zur Rolle von Unternehmen in einer Transformation hin $\mathrm{zu}$ einer Kreislaufwirtschaft in den letzten Jahren deutlich zugenommen. Neben dem Fokus auf recyclingorientierten Maßnahmen, die eher nachsorgend wirken, sind auch proaktive Ansatzpunkte für eine effektive Transformation hin $\mathrm{zu}$ einer nachhaltigen Kreislaufwirtschaft entscheidend, dies betrifft insbesondere die Adaption des Geschäftsmodells eines Unternehmens (Santa-Maria et al. 2021) oder das Design der Produkte und Dienstleistungen eines Unternehmens (Bocken et al. 2016). Das Ziel einer Kreislaufwirtschaft besteht in der Reduktion von Umweltauswirkungen. Um dies zu erreichen ist die Geschwindigkeit des Durchsatzes von Stoffströmen in der Gesellschaft $\mathrm{zu}$ verlangsamen und es sind Stoffkreisläufe zu schließen, wodurch es $\mathrm{zu}$ einem geringeren Ressourceninput in den gesellschaftlichen Metabolismus und $\mathrm{zu}$ weniger Abfällen kommen sollte (Bocken et al. 2016; Geissdoerfer et al. 2018). Häufig wird eine Kreislaufwirtschaft mit einer verbesserten Abfallwirtschaft und einem umfassenden Recycling assoziiert (Schöggl et al. 2020b). Allerdings muss eine Kreislaufwirtschaft ganzheitlicher gesehen werden, womit über das Recycling hinausgehende R-Strategien, die einer hierarchischen Reihung folgen, in den Fokus rücken; hier sind insbesondere die "10-R“ Strategien von Reike et al. (2018) zu nennen: 1) refuse, 2) reduce, 3) reuse, 4) repair, 5) refurbish, 6) remanufacture, 7) repurpose, 8) recycle, 9) recover und 10) remine.

Während insgesamt konzeptionelle Studien die wissenschaftliche Diskussion zur Kreislaufwirtschaft dominieren (Govindan und Hasanagic 2018), sind nun zunehmend auch empirische Studien, die sich auf die Umsetzung im Unternehmenskontext fokussieren (Stumpf et al. 2021), zu finden. Eine weitere Studie von Bocken et al. (2017) untersuchte beispielsweise, wie multinationale Unternehmen die Kernideen einer Kreislaufwirtschaft in ihre Kommunikation integriert haben und in den berichteten Geschäftsaktivitäten positionieren. Mithilfe einer automatisierten Inhaltsanalyse einer Untergruppe von Standard \& Poor's (S\&P) 500 börsennotierten Firmen aus einer Datenbank mit Unternehmenspressemitteilungen im Zeitraum von 2005 bis 2014 konnte festgestellt werden, dass die häufigsten Keywords Recycling, Instandhaltung (maintenance), Refurbishing sowie Abfallmanagement waren. Neben solchen sekundärdatenbasierten Analysen, Fallstudien und statistischen Modellen, widmet sich eine zunehmende Anzahl an Arbeiten der Erhebung vom Primärdaten in verschiedenen Ländern. So analysieren etwa Mura et al. (2020) die Umsetzung einer Kreislaufwirtschaft in italienischen klein- und mittelständischen Unternehmen (KMUs) hinsichtlich Er- folgsfaktoren und Zusammenhängen mit Unternehmensstrategien und -erfolg. Walker et al. (2021) vergleichen Industrieperspektiven zu Aspekten der sozialen Nachhaltigkeit einer Kreislaufwirtschaft in den Niederlanden und Italien. Ormazabal et al. (2018) erheben Hindernisse und Chancen einer Kreislaufwirtschaft in spanischen KMUs.

Dem generellen Trend der Literatur folgend hat in den letzten Jahren auch die Anzahl an Studien zugenommen, die sich der Kreislaufwirtschaft in Österreich widmen. Darunter liefern Holzer et al. (2021) eine erste sektorübergreifende empirische Erhebung mit 183 österreichischen KMUs, in welcher sechs relevante Themenbereiche und Möglichkeiten der Umsetzung einer Kreislaufwirtschaft in KMUs aufgezeigt werden. Von diesen sechs Themenbereichen werden in einer „Performance-Importance“ Messung neben den Aspekten „Stakeholder Kooperation“, „Unabhängigkeit von Ressourcenverfügbarkeiten“ und „Kenntnis des Produktlebenszyklus" die Themen "Nachhaltigkeit“, „Ressourceneffizienz“ und „Differenzierung“ als am wichtigsten eingeschätzt. Während diese Studie spezifische Handlungsmöglichkeiten zur Transformation in Richtung Kreislaufwirtschaft österreichischer KMUs identifiziert, gibt es bis dato noch keine empirische Studie, die sich dem Umsetzungsgrad solcher Handlungsmöglichkeiten einer nachhaltigen Kreislaufwirtschaft in österreichischen Unternehmen widmet. Diese Forschungslücke wird im vorliegenden Beitrag durch die Beantwortung der folgenden drei Forschungsfragen (FF) adressiert:

FF1: Wie sieht der Umsetzungsgrad verschiedener nachhaltiger und zirkulärer Produktmanagementpraktiken in österreichischen Produktionsunternehmen aus?

FF2: Wie nehmen österreichische GeschäftsführerInnen die Auswirkungen nachhaltiger und zirkulärer Produktmanagementpraktiken auf die Nachhaltigkeitsleistung und den Unternehmenserfolg wahr?

FF3: Wie schätzen GeschäftsführerInnen das Marktpotenzial für die Kreislaufwirtschaft in Österreich ein?

Zur Beantwortung dieser Forschungsfragen wurden Telefoninterviews mit 120 GeschäftsführerInnen und 100 Nachhaltigkeitsverantwortlichen österreichischer Unternehmen durchgeführt. Der Fokus lag auf Unternehmen aus dem produzierenden Gewerbe. Die 
Tab. 1 Verwendete Skalen und Quellen

\begin{tabular}{|c|c|c|c|c|}
\hline Konstrukt & Fragen & Antworten & Interviewpartner & Quelle(n) \\
\hline Nachhaltigkeitsmanagement generell & 10 & 100 & Führungskräfte & Selbst erstellt \\
\hline Kreislaufwirtschaftsaktivitäten & 16 & 100 & Führungskräfte & $\begin{array}{l}\text { (Ellen MacArthur Foundation 2015; Khan et al. 2020; } \\
\text { Reike et al. 2018; Schöggl et al. 2017) }\end{array}$ \\
\hline Verfügbarkeit von Recyclingdaten & 2 & 100 & Führungskräfte & Selbst erstellt \\
\hline Strategische Ausrichtung auf eine Kreislaufwirtschaft & 13 & 100 & Führungskräfte & (Ellen MacArthur Foundation 2020) \\
\hline $\begin{array}{l}\text { Effekte von Nachhaltigkeits- und Kreislaufwirtschaftsaktivi- } \\
\text { täten auf die Nachhaltigkeitsleistung }\end{array}$ & 10 & 120 & $\begin{array}{l}\text { Geschäftsfüh- } \\
\text { rerlnnen }\end{array}$ & (Rauter et al. 2019) \\
\hline $\begin{array}{l}\text { Effekte von Nachhaltigkeits- und Kreislaufwirtschaftsaktivi- } \\
\text { täten auf die Nachhaltigkeitsleistung }\end{array}$ & 7 & 120 & $\begin{array}{l}\text { Geschäftsfüh- } \\
\text { rerlnnen }\end{array}$ & (Rauter et al. 2019) \\
\hline $\begin{array}{l}\text { Einschätzung der Kreislaufwirtschaftsbereitschaft des } \\
\text { Marktes }\end{array}$ & 7 & 120 & $\begin{array}{l}\text { Geschäftsfüh- } \\
\text { rerlnnen }\end{array}$ & (Khan et al. 2020) \\
\hline
\end{tabular}

Interviewfragen wurden aus der Literatur abgeleitet und bauen auf etablierten Konzepten wie den 10-Rs (Reike et al. 2018) oder dem Rahmenwerk der ReSOLVE $^{1}$ Strategien (Ellen MacArthur Foundation 2015) auf.

Dieser Beitrag ist wie folgt strukturiert: in Kapitel 2 erfolgt die Diskussion der theoretischen Grundlagen, insbesondere wird auf die Rolle des Recyclings in einer Kreislaufwirtschaft sowie auf die Ausgangslage hinsichtlich Kreislaufwirtschaft in Österreich eingegangen. In Kapitel 3 wird die methodische Vorgehensweise (Erarbeitung der Fragebogenkonstrukte, Durchführung der Telefoninterviews) beschrieben. Anschließend werden in Kapitel vier die Ergebnisse der empirischen Studie dargestellt. Dieses Kapitel unterteilt sich in fünf Abschnitte, die auf unterschiedliche relevante Themen eingehen: (1) Nachhaltigkeitsmanagement, (2) Nachhaltiges und kreislaufwirtschafts-orientiertes Produktmanagement, (3) strategische Ausrichtungen, (4) Auswirkungen von Nachhaltigkeitsund Kreislaufwirtschaftsaktivitäten auf die Nachhaltigkeitsleistung und den Unternehmenserfolg sowie (5) Einschätzung des österreichischen Marktes im Kontext einer Kreislaufwirtschaft. In Kapitel fünf werden die Ergebnisse diskutiert und in Kapitel sechs wird das Fazit dargelegt.

\footnotetext{
${ }^{1}$ Regenerate/Regenerieren, Share/Teilen, Optimize/Optimieren, Loop/im Kreislauf führen, Virtualize/Virtualisieren, Exchange/Austauschen.
}

\section{Theoretische Fundierung}

\subsection{Recycling im Kontext einer} Kreislaufwirtschaft

Von 1970 bis 2017 hat sich die globale Rohstoffgewinnung pro Jahr mehr als verdreifacht und nimmt weiter $\mathrm{zu}$ (International Resource Panel 2019). Dieser enorme Anstieg an geförderten Rohstoffen führt zu hohen Treibhausgasemissionen, denn etwa die Hälfte der gesamten globalen Treibhausgasemissionen und mehr als $90 \%$ des Biodiversitätsverlusts sind auf die Gewinnung und Verarbeitung von Rohstoffen, Kraftstoffen und Lebensmitteln zurückzuführen (ibid.). Bei näherer Betrachtung der Treibhausgasemissionen in der EU zeigt sich, dass etwa $20 \%$ auf die Industrie zurückzuführen sind (European Commission 2019). Das industrielle Handeln ist nach wie vor sehr „linear“ und abhängig vom Durchsatz an neuen Materialien, die gefördert, gehandelt und $\mathrm{zu}$ Waren verarbeitet und schließlich als Abfall oder Emissionen entsorgt werden (ibid.). Nur etwa $12 \%$ der industriell verwendeten Materialien stammen aus Recyclingquellen (ibid.). Die EU geht im „European Green Deal“ näher auf diese Herausforderungen ein und setzt sich zum Ziel, eine gerechtere und prosperierende Gesellschaft mit einer modernen, ressourceneffizienten und wettbewerbsfähigen Wirtschaft zu schaffen, in der es im Jahr 2050 keine Nettoemissionen von Treibhausgasen gibt und in der das Wirtschaftswachstum vom Ressourcenverbrauch entkoppelt ist. Um den primären Rohstoffabbau zu minimieren, wird die Nutzung von „Abfällen“ als Ressource unerlässlich werden (European Commission 2019).

Vor etwa drei Jahrzehnten, im Jahr 1990, wurde die Kreislaufwirtschaft mit ihrem 3R-Prinzip vorgestellt: Reduzie- rung, Wiederverwendung und Recycling von Materialien, Abfällen und auch Energie (Heshmati 2015). Das „R“ steht für die verschiedenen Begriffe, die mit „re-“ beginnen, was im Lateinischen „wieder“ bedeutet, z. B. re-duce, re-use, re-cycle (Reike et al. 2018). Die R-Strategien sind nuancierte Materialhierarchien zur Operationalisierung von kreislaufwirtschaftlichen Prinzipien, die den Werterhalt von Materialien über viele Produktlebenszyklen ermöglichen sollen (ibid.). Auf Produktebene können die Kreislaufwirtschaft und die R-Prinzipien wirkungsvolle Ansätze sein, um die Art und Weise, wie Organisationen ihr Ressourcenmanagement betreiben und verwalten, zu gestalten und sie zu inspirieren, den gesamten Produktlebenszyklus zu überdenken (ibid.).

Mit der Popularisierung des Konzepts der Kreislaufwirtschaft in Forschung und Gesellschaft stellt sich auch die Frage, welchen Beitrag Ansätze einer Kreislaufwirtschaft zur Erreichung von nachhaltigen Entwicklungszielen (wie etwa formalisiert in den Sustainable Development Goals (SDGs)) leisten können. In der wissenschaftlichen Literatur bildet sich diesbezüglich zunehmender Konsens dazu, dass eine Kreislaufwirtschaft als eine von mehreren komplementären Strategien zur Erreichung von nachhaltiger Entwicklung gesehen werden sollte (Corvellec et al. 2021; Geissdoerfer et al. 2017; Schöggl et al. 2020b), da eine Kreislaufwirtschaft nicht alle Nachhaltigkeitsthemen adressiert (Cecchin et al. 2021) und da kreislaufwirtschaftliche Aktivitäten nicht zwingend die negativen ökologischen und sozialen Auswirkungen von Produktions- und Konsumsystemen reduzieren (Zink und Geyer 2017) oder es gar zu Rebound Effekten kommen könnte (Figge und Thorpe 2019). 


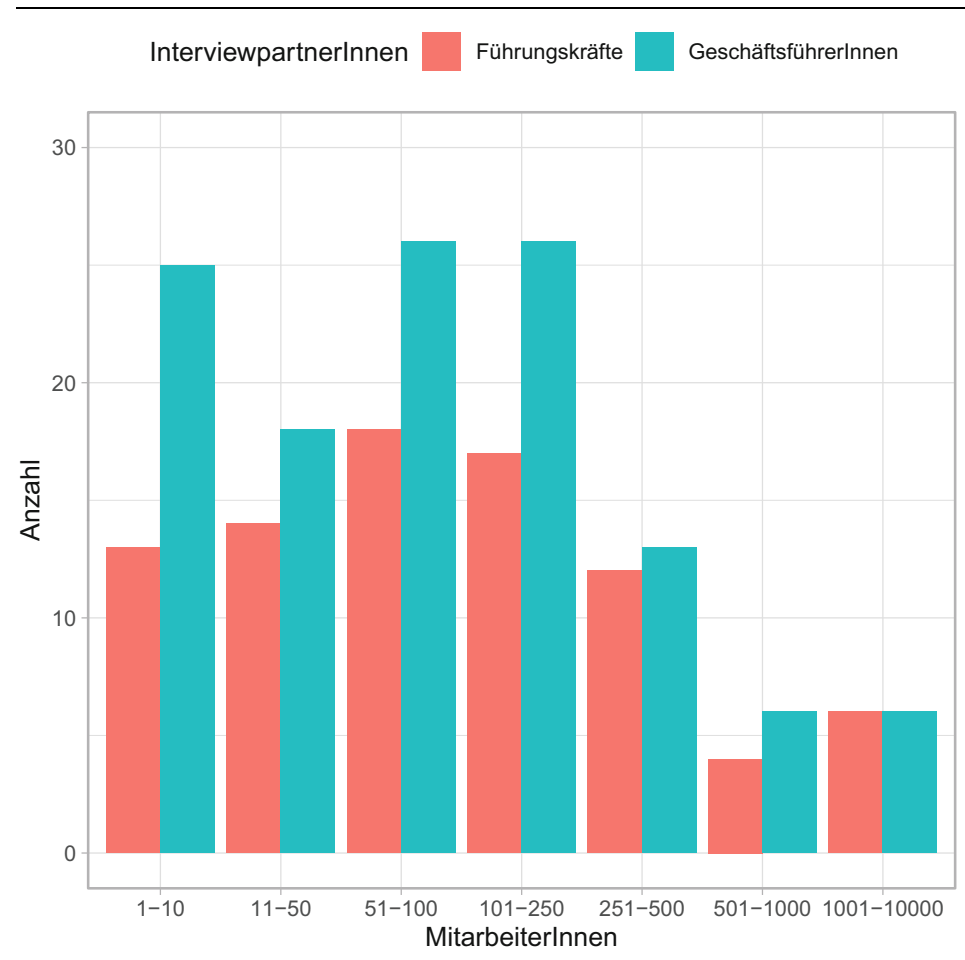

Abb. 1 Anzahl an MitarbeiterInnen der befragten Unternehmen. Führungskräfte ( $n=100)$, GeschäftsführerInnen $(n=120)$

Aus diesem Grund wird diesem Artikel auch ein „nachhaltiges“ Verständnis einer Kreislaufwirtschaft zugrunde gelegt, entsprechend welchem der Fokus der empirischen Erhebung auf nachhaltigem Produktmanagement liegt und neben Ansätzen zur Schließung von Material- und Stoffkreisläufen auch soziale und weitere ökologische Managementpraktiken erhoben wurden.

\subsection{Kreislaufwirtschaft in Österreich}

Allgemein ist Österreich noch am Anfang seiner Entwicklung im Bereich Kreislaufwirtschaft. Gleichzeitig ist die Entwicklung auf legislativer Ebene sehr dynamisch. Als Grundlage für die Kreislaufwirtschaft kann in Österreich das Abfallwirtschaftsgesetz 2002 (Fassung: Oktober 2021) (Bundesgesetz über eine nachhaltige Abfallwirtschaft 2021) gesehen werden. Weitere Verordnungen umfassen einzelne Behandlungsschritte des Abfalls (z.B. Deponieverordnung 2021) oder bestimmte Stoffströme (z.B. Recycling-Baustoffverordnung 2015; Verpackungsverordnung 2014) sowie die Ökodesign-Verordnung (Ökodesign-Verordnung 2011). Besonders im Teilbereich Recycling schneidet das Land im internationalen Vergleich gut ab (Tschulik 2021). Dies wurde auch
2019 im „Circularity Gap“ Bericht festgestellt, dem ersten seiner Art auf nationaler Ebene (Circle Economy und ARA 2019), wo ein Zirkularitätsindex von $9,7 \%$ ausgewiesen wurde. Dieser Indikator beschreibt den „Anteil von Sekundärrohstoffen im Gesamtverbrauch von Materialien " (Circle Economy und ARA 2019). Ebenfalls 2019 wurde der Masterplan Umwelttechnologie (MUT) veröffentlicht, der den Aufbruch in die Kreislaufwirtschaft als eines der zentralen vier Themen definiert. Zentrale Maßnahmen des MUT im Zeitraum 2019-2030 sollen eine Forcierung der Kreislaufwirtschaft durch Digitalisierung sowie eine verbesserte Förderung von Sammel-, Sortier- und Recyclingtechnologien sein. So sollen vorrangig Projekte unterstützt werden, die die „Digitalisierung entlang der abfallwirtschaftlichen Wertschöpfungskette umsetzen" (Bundesministerium für Nachhaltigkeit und Tourismus 2019, S. 48). Auch Aspekte, die mit ganzheitlichem Produktmanagement (Life Cycle Management) in Verbindung stehen, wie Design für Nachhaltigkeit, Verlängerung von Produktlebensdauer, oder ein stärkerer Fokus auf die Reparierfähigkeit von Produkten werden bereits angesprochen, wenn auch nicht mit konkreten Maßnahmen hinterlegt. Im
März 2021 wurde schließlich mit der Erarbeitung einer Strategie zur Forcierung der Kreislaufwirtschaft in Österreich begonnen (Tschulik 2021). Die Einhaltung der planetaren Grenzen sowie der große Handlungsbedarf bei der Reduktion des Verbrauchs von Primärrohstoffen spielen hierbei eine besonders wichtige Rolle (ibid.). Eine Fertigstellung der Strategie ist für Ende 2021 vorgesehen, mit ersten Umsetzungsschritten soll Anfang 2022 begonnen werden.

\section{Methodische Vorgehensweise}

Die insgesamt 220 strukturierten Telefoninterviews, die für diese Studie deskriptiv ausgewertet wurden, stützten sich auf Fragen, welche großteils aus der wissenschaftlichen Literatur abgeleitet oder direkt aus etablierten Konstrukten übernommen wurden. Tab. 1 bietet eine Übersicht über die verwendeten Skalen und deren Quellen. Im Zuge der Interviews wurden 100 Führungskräfte mit Verantwortung zu Nachhaltigkeits- und Kreislaufwirtschaftsaktivitäten und 120 GeschäftsführerInnen von österreichischen Produktionsunternehmen befragt. Insgesamt 84 dieser kamen aus denselben Unternehmen (d.h. sowohl die GeschäftsführerInnen als auch die Nachhaltigkeitsverantwortlichen eines Unternehmens haben geantwortet). Auf diese Doppelbefragung, welche mit Hinsicht auf die Erstellung von statistischen Modellen durchgeführt wurde, wird im Zuge der vorliegenden deskriptiven Analyse nicht weiter eingegangen. Die beiden Datensätze werden getrennt und jeweils für sich allein analysiert. Insgesamt wurden 171 produzierende Unternehmen kontaktiert, welche zufällig aus einem Unternehmensregister ausgewählt wurden.

Abb. 1 zeigt die Anzahl der MitarbeiterInnen der befragten Unternehmen. Aufgrund der angestrebten, aber nicht immer erfolgreichen Doppelbefragung von Führungskräften $(n=100)$ und GeschäftsführerInnen $\quad(n=120)$ derselben Unternehmen, unterscheidet sich die Anzahl, wie ersichtlich, teilweise. Abb. 2 zeigt die Verteilung der Unternehmen je nach Sektor $(n=17)$ mit derselben Unterscheidung von Führungskräften und GeschäftsführerInnen wie bei Abb. 1 . 


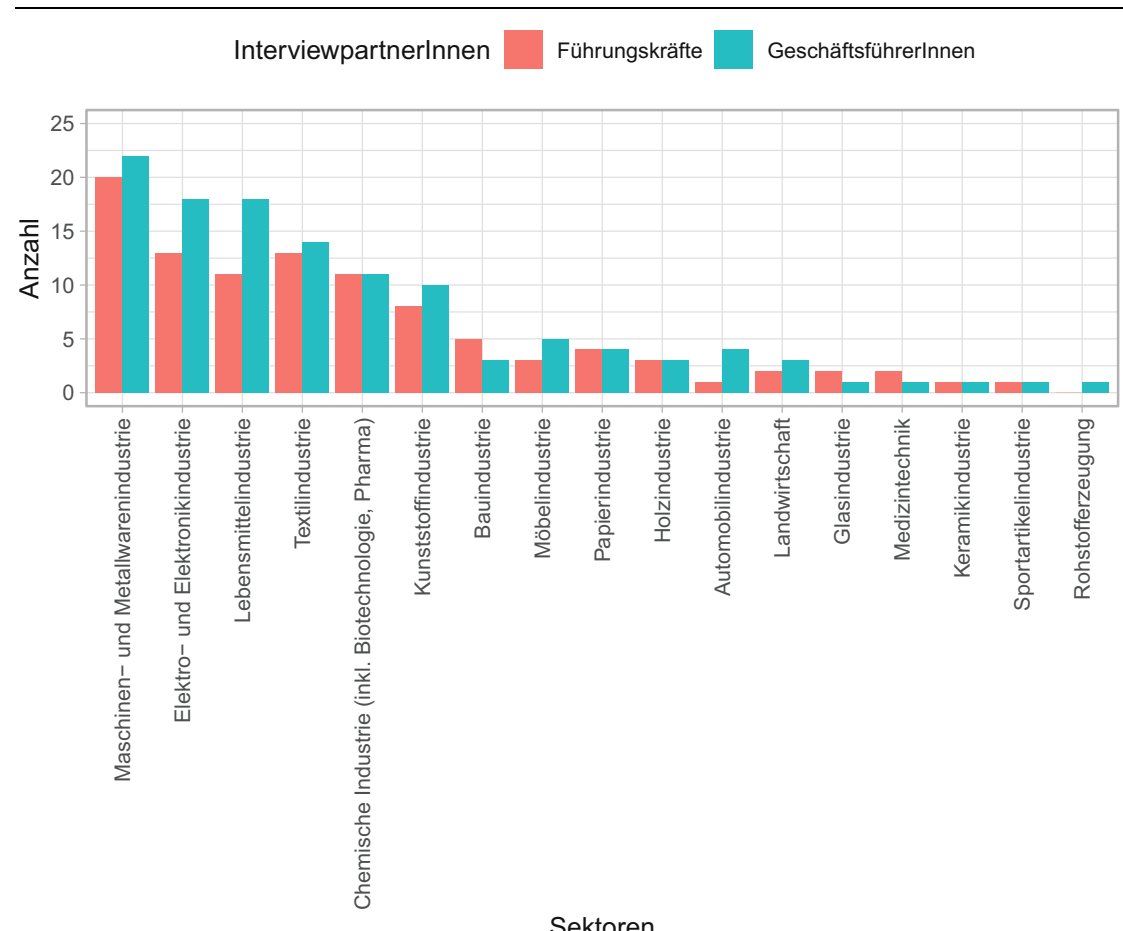

Abb. 2 Verteilung der Unternehmen je Sektor. Führungskräfte $(n=100)$, Geschäftsführerlnnen $(n=120)$

\section{Nachhaltigkeitsmangement}

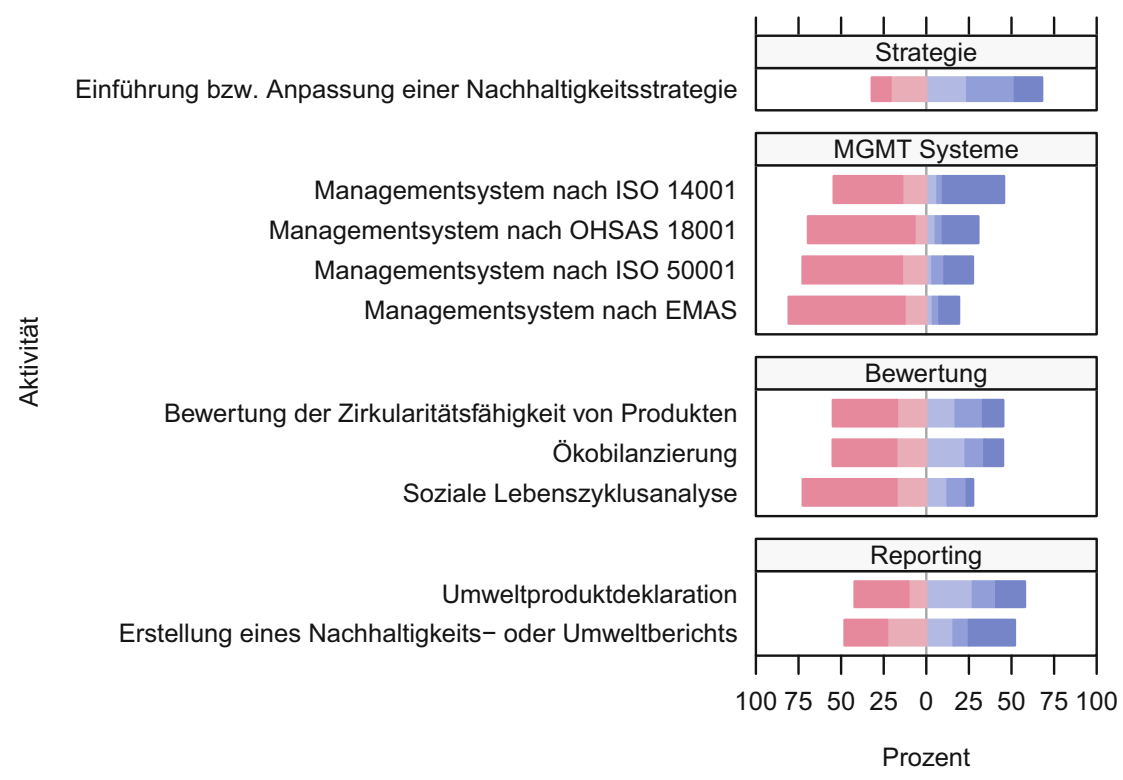

Abb. 3 Nachhaltigkeitsmanagementpraktiken in österreichischen Produktionsunternehmen ( $n=100$ Führungskräfte); Balkenfarben von links nach rechts: $r o t-n i c h t$ vorhanden; hellrot -ziehen es in Betracht; hellblau -in Einzelfällen zutreffend; blau - unternehmensweite Umsetzung im Gange; dunkelblau - bereits unternehmensweit umgesetzt

\section{Ergebnisse}

In diesem Kapitel werden die Ergebnisse der empirischen Befragung zusammengefasst. Dabei wird in den Abschn. 4.1-4.3 auf die Antworten der Führungskräfte zum Implementierungsgrad von Nachhaltigkeits- und Kreislaufwirtschaftspraktiken auf Unternehmensebene sowie die strategische Ausrichtung der Unternehmen eingegangen. Abschn. 4.4 und 4.5 fassen die Einschätzungen der GeschäftsführerInnen zum Effekt von Nachhaltigkeits- und Kreislaufwirtschaftspraktiken auf die Nachhaltigkeitsleistung und den ökonomischen Erfolg ihrer Unternehmen und dem Marktpotential einer Kreislaufwirtschaft in Österreich zusammen.

\subsection{Nachhaltigkeitsmanagement in österreichischen Unternehmen}

Um eine Einschätzung des Umsetzungsgrades von verschiedenen Ansätzen des Nachhaltigkeitsmanagements in österreichischen Unternehmen $\mathrm{zu}$ erhalten, wurden den Führungskräften zu Beginn Fragen hinsichtlich des Vorhandenseins einer allgemeinen Nachhaltigkeitsstrategie sowie zu nachhaltigkeitsbezogenen Managementsystemen und Nachhaltigkeitsbewertungsund -berichtsansätzen gestellt. Wie in Abb. 3 ersichtlich, weisen Umweltmanagementsysteme nach ISO 140001 (35\%) den höchsten unternehmensweiten Implementierungsgrad auf, gefolgt von der Veröffentlichung von Nachhaltigkeitsberichten $(27 \%)$ und der Implementierung von Arbeitsschutzmanagementsystemen nach OHSAS 18001 (20\%).

Berücksichtigt man zusätzlich auch noch sich im Gange befindliche unternehmensweite Umsetzungen und erste Umsetzungen in Pilotprojekten (also alle drei blauen Balken in Abb. 3), so zeigt sich, dass mehr als zwei Drittel (68\%) der befragten Unternehmen zumindest teilweise eine Nachhaltigkeitsstrategie definiert haben und jeweils etwas mehr als die Hälfte Umweltproduktdeklarationen $(57 \%)$ oder Nachhaltigkeitsberichte $(52 \%)$ verfasst haben. Den geringsten Implementierungsgrad weisen Umweltmanagementsysteme nach EMAS (19\%), Energiemanagementsysteme nach ISO 50001 (27\%) und die Durchführung von sozialen Lebenszyklusanalysen (SLCAs) auf (27\%). In der Kategorie der Produktbewertung zeigt 


\section{Nachhaltigkeits- und Kreislaufwirtschaftsaktivitäten in österreichischen Produktionsunternehmen}

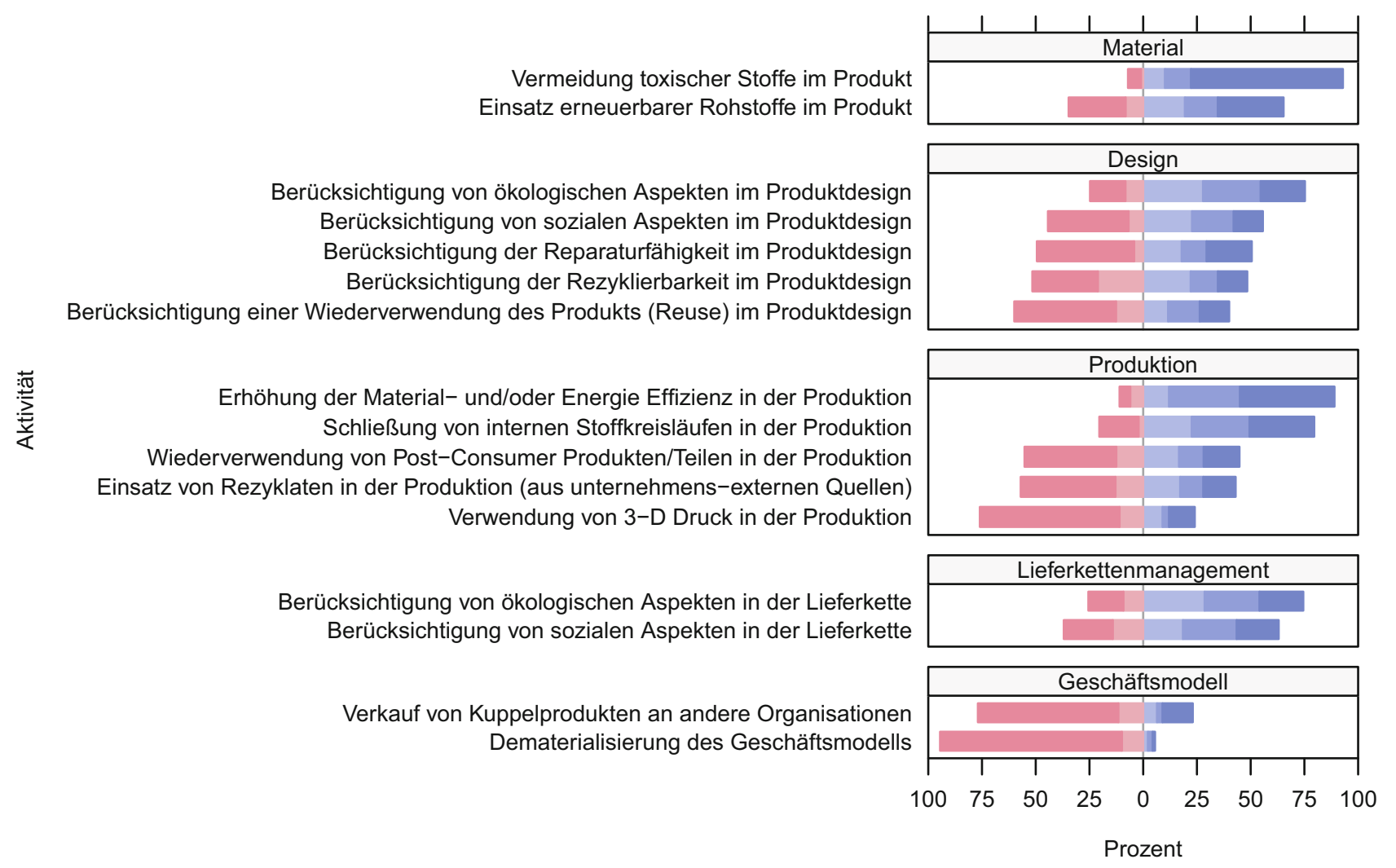

Abb. 4 Nachhaltigkeits- und Kreislaufwirtschafsaktivitäten in österreichischen Produktionsunternehmen ( $n=100$ Führungskräfte); Balkenfarben von links nach rechts: rot - nicht vorhanden; hellrot - ziehen es in Betracht; hellblau - in Einzelfällen zutreffend; blau unternehmensweite Umsetzung im Gange; dunkelblau - bereits unternehmensweit umgesetzt

sich, dass der unternehmensweite Implementierungsgrad von Bewertungsansätzen generell noch gering ist, vor allem hinsichtlich sozialer Aspekte der Nachhaltigkeit, sich aber jeweils knapp die Hälfte der Unternehmen zumindest in Pilotprojekten Lebenszyklusanalysen (LCA) und Zirkularitätsbewertungen gewidmet haben.

\subsection{Ansätze des nachhaltigen und kreislaufwirtschafts-orientieren Produktmanagements}

Einen tieferen Einblick in die Umsetzung von Nachhaltigkeits- und Kreislaufwirtschaftsaktivitäten auf operativer Ebene lieferte die Auswertung der Frage zum Umsetzungsgrad von 16 spezifischen kreislaufwirtschaftlichen Praktiken (Abb. 4). Die abgefragten Praktiken können in fünf Kategorien unterteilt werden, in welchen jeweils mehrere Aspekte zusammengefasst wurden. Die Aspekte wurden in den Kategorien (i) Material, (ii) Design, (iii) Produktion, (iv) Lieferkettenmanagement und (v) Geschäftsmodell thematisch gruppiert. Das Spektrum umfasst sowohl relevante Themen der Kreislaufwirtschaft (z. B. die R-Prinzipien (Reike et al. 2018) oder das ReSOLVE-Rahmenwerk (Ellen MacArthur Foundation 2015)) als auch Themen, die für die Berücksichtigung eines umfassenderen Nachhaltigkeitsmanagements relevant sind (z.B. soziale und ökologische Auswirkungen entlang der Lieferkette).

Die Auswertung zeigt, dass die Vermeidung von toxischen Stoffen in Produkten und eine erhöhte Material- und/ oder Energieeffizienz in der Produktion, die bereits am häufigsten unternehmensweit umgesetzten Aspekte sind. Dieses Ergebnis lässt vermuten, dass kostengetriebene und operative Faktoren eine höhere Umsetzungsrelevanz in Unternehmen haben als strategische Faktoren wie z. B. "Design for X“ in der Produktentwicklung. Weitere häufig umgesetzte Themen sind das umweltgerechte/ökologische Design von Produkten, die Schließung interner Stoffkreisläufe in der Produktion, ein grünes und soziales Lieferkettenmanagement sowie die Verwendung erneuerbarer Ressourcen für Produkte. Allerdings werden diese Aspekte bisher meist nur in Pilotprojekten umgesetzt oder die unternehmensweite Umsetzung ist im Gange. Erst in wenigen Firmen sind diese Aspekte bereits unternehmensweit umgesetzt.

Die recyclingorientierte Gestaltung von Produkten („Design for recycling“) wird bereits von knapp der Hälfte (48\%) der Unternehmen ganz oder teilweise umgesetzt. Ein weiterer interessanter Aspekt ist, dass rund $43 \%$ der befragten Unternehmen teilweise oder umfassend recycelte Materialien in ihrer Produktion einsetzen oder das Wiederverwenden von „post-consumer“ Gütern berücksichtigen bzw. in Betracht ziehen (45\%). 


\section{Datenverfügbarkeit}

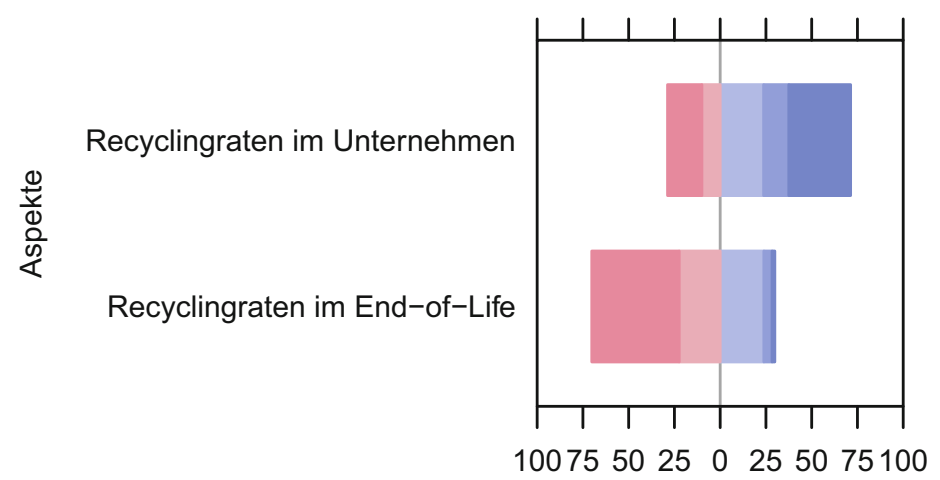

Prozent

Abb. 5 Verfügbarkeit von Daten ( $n=100$ Führungskräfte); Balkenfarben von links nach rechts: rot - gar nicht verfügbar; hellrot - kaum verfügbar; hellblau teilweise verfügbar; blau-zum Großteil verfügbar; dunkelblau -vollständig verfügbar

Die Aspekte, die bisher von vielen der befragten Unternehmen noch gar nicht berücksichtigt wurden, sind die Verwendung von 3D-Druckern als mögliche Form der flexiblen, dezentralen Produktion von Teilen die z.B. nur in kleinen Stückzahlen benötigt werden und auch beide Aspekte, die in das Geschäftsmodell der Firmen eingreifen würden. Eine Möglichkeit, um die Kreislaufwirtschaft voranzutreiben, wäre die Entwicklung von dematerialisierten Geschäftsmodellen. Auch der Verkauf von Nebenerzeugnissen könnte zu einer Abfallreduktion führen. Allerdings war bei den beiden letztgenannten Aspekten auch der Anteil der fehlenden Antworten am höchsten, was ein Indiz für die Kontextspezifität dieser Ansätze sein könnte.

Es zeigt sich, dass die Kreislaufwirtschafspraktiken in österreichischen Produktionsunternehmen noch eher auf Compliance und Effizienzthemen fokussiert sind. Veränderungen auf der Ebene von Geschäftsmodellen werden von den Unternehmen noch seltener angestrebt. Im Bereich des Lieferkettenmanagements spielt jedoch die Integration von nachhaltigen/grünen und sozialen Aspekten bereits bei den meisten Unternehmen eine wichtige Rolle bzw. wurde hier schon in vielen Fällen die unternehmensweite Umsetzung vorangetrieben.

Im Zuge der Befragung wurde in verschiedenen Fragekategorien speziell auf recycling-relevante Aspekte eingegangen. Abb. 5 zeigt diesbezüglich die Einschätzung der Führungskräfte zur
Verfügbarkeit von recycling-bezogenen Daten. Wie in der Grafik ersichtlich geben etwas weniger als die Hälfte der Führungskräfte $(47 \%)$ an, dass sie über Daten zu unternehmensinternen Reyclingraten großteils oder vollständig verfügen. Ein weiteres Viertel (24\%) gibt an, diese Daten zumindest teilweise zur Verfügung zu haben. Im Gegensatz dazu ist das Wissen über Verwertungswege im End-of-Life der Produkte noch stark begrenzt. Während ebenfalls ein Viertel angab, teilweise über Recyclingraten im End-of-Life informiert zu sein, so hatten diese Informationen nur $4 \%$ großteils und sogar nur $1 \%$ vollständig. Dieses fehlende Wissen offenbart einen beträchtlichen Aufholbedarf hinsichtlich der Schließung von Informationslücken zwischen der Beginningof-Life und der End-of-Life Phase von Produkten. Diese ist eine wichtige Voraussetzung für ein effektives closedloop Recycling und die Umsetzung von kreislaufwirtschaftlichem Design- oder Geschäftsmodellstrategien.

\subsection{Strategische Ausrichtung auf eine Kreislaufwirtschaft}

Die strategische Orientierung von Unternehmen im Bereich der Kreislaufwirtschaft umfasst verschiedene Ebenen und beinhaltet eine strategische Ausrichtung an den Prinzipien der Kreislaufwirtschaft. Die Ebenen, nach denen in der Umfrage unterschieden wurden, sind (i) Strategie und Planung, (ii) kreislaufwirtschaftliche Innovation, (iii) Humankapital sowie (iv) Betrieb.
Abb. 6 stellt diese vier unterschiedlichen Ebenen inklusive der einzelnen Ausprägungen und Ergebnisse dar. Im Bereich Strategie und Planung wird deutlich, dass sich bereits viele Unternehmen intern mit der Thematik Kreislaufwirtschaft auseinandersetzen oder bereits eine eigene Umsetzung planen oder durchgeführt haben. So stimmen beispielsweise $64 \%$ der Befragten eher oder ganz der Aussage $\mathrm{zu}$, dass Kreislaufwirtschaft zumindest in einzelnen Unternehmensbereichen oder Projekten eine Rolle in der strategischen Agenda spielt. Auch bei den Themen Risikomanagement und Anlehnung der Gesamtstrategie an das Konzept einer Kreislaufwirtschaft sowie bei der Definition messbarer kreislaufwirtschaftlicher Ziele (wie Recyclingraten) gaben mehr als die Hälfte der Befragten an, dass bereits an einer konkreten Umsetzung gearbeitet werde. Gleichzeitig wird deutlich, dass Unternehmen ihre Zielsetzungen und strategischen Überlegungen nicht öffentlich verfügbar machen wollen. Wie in Abb. 6 sichtbar wird, gibt hier eine große Mehrheit (81\%) der Befragten an, dass es für das Unternehmen keine öffentlich verfügbaren Dokumente zu diesen Fragen gibt, während in $84 \%$ der Fälle auch keine öffentlich verfügbaren Ziele im Bereich der Kreislaufwirtschaft vorhanden waren. Diese Diskrepanz verdeutlicht, dass kreislaufwirtschaftliche Themen intern bei Unternehmen eine Rolle spielen, diese aber (noch) nicht in eine öffentliche Kommunikation oder belastbare Ziele gegossen werden, wie dies beispielsweise bei der Erstellung von Klimastrategien immer häufiger der Fall ist.

Kreislaufwirtschaftliche Innovation wurde abgefragt anhand der Fähigkeit der Unternehmen in dem Bereich zu innovieren. Wichtige Aspekte dieser Fähigkeiten konnten beispielsweise Mitgliedschaften in Forschungsprojekten, strategische Allianzen, oder auch die Aufstellung des Innovationsmanagements im Allgemeinen sein. Kreislaufwirtschaftliche Innovation beinhaltete sowohl zirkuläre Geschäftsmodelle, Produkte, als auch Services. Von dieser Fähigkeit ist nur eine Minderheit der Unternehmen überzeugt (ca. die Hälfte der Befragten hält die eigenen Fähigkeiten für nicht ausreichend, weitere $27 \%$ nur in Einzelfällen für ausreichend). Dies kann bedeuten, dass sich Unternehmen für eine produktive Umsetzung kreislaufwirtschaftlicher Strategien und 


\title{
Strategische Ausrichtung
}

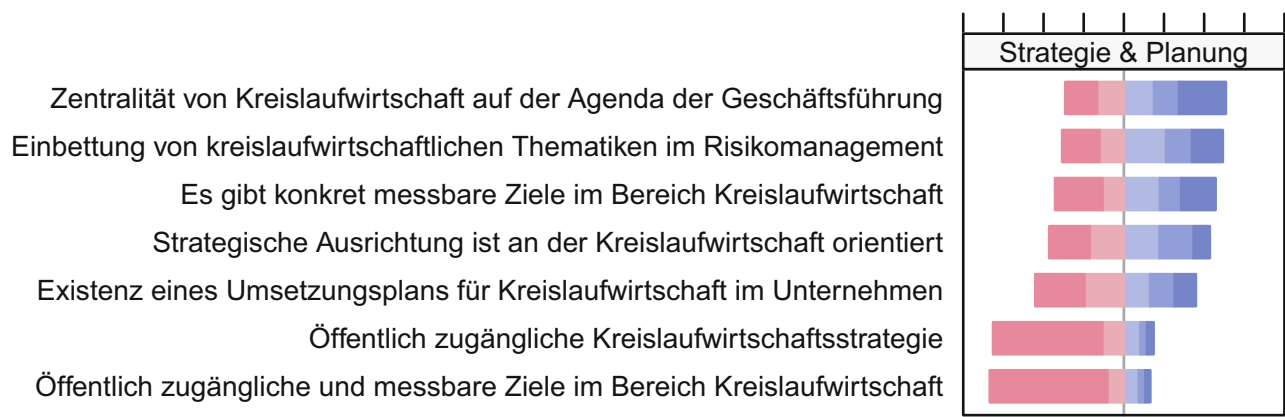

Ausmaß der Ausrichtung der Innovationskapazität an der Schaffung zirkulärer Ansätze*

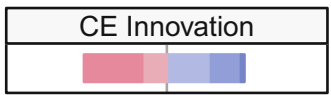

\begin{abstract}
Interne Kommunikation einer Kreislaufwirtschatsstrategie sowie Aktivitäten, diese zu implementieren
\end{abstract} Kreislaufwirtschaftlich orientierte Weiterbildungsangebote innerhalb des Unternehmens

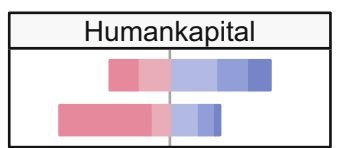

\begin{abstract}
Wir besitzen geeignete Werke, Anlagen und Eigentum um zirkuläre Ansätze* zu unterstützen Existenz von Prozessen, um zirkuläre Ansätze* zu unterstützen

Verfügbarkeit geeigneter IT und anderer digitaler Systeme um zirkuläre Ansätze* zu unterstützen
\end{abstract}

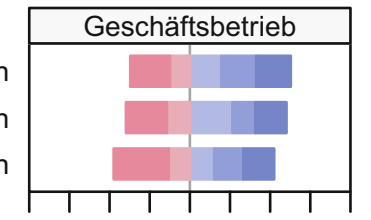

1007550250255075100

Prozent

Abb. 6 Strategische Ausrichtung an den Prinzipien der Kreislaufwirtschaft ( $n=100$ Führungskräfte); Balkenfarben von links nach rechts:rot-nichtvorhanden; hellrot-ziehenes inBetracht; hellblau-inEinzelfällenzutreffend; blau-unternehmensweiteUmsetzung im Gange; dunkelblau - bereits unternehmensweit umgesetzt; *zirkuläre Geschäftsmodelle, Produkte und Services

Prinzipien neuen, bisher wenig erprobten Vorgehensweisen zur Innovation öffnen müssen. Dies kann beispielsweise die Umsetzung von Innovationsprojekten mit Partnern bedeuten, mit denen zuvor keine Erfahrungen gesammelt wurden.

Eine weitere Maßnahme zur Erhöhung der Innovationskapazität für die Kreislaufwirtschaft ist im Bereich der Humanressourcen zu sehen. Hierbei gibt ein Großteil der Unternehmen an, MitarbeiterInnen über Kreislaufwirtschaft und die Umsetzung dieser im Unternehmen zu informieren (beispielsweise durch Broschüren, aber auch Webinare, Konferenzen, etc.). So können MitarbeiterInnen mit dem Thema vertraut gemacht werden. Weniger aktiv sind Unternehmen darin, MitarbeiterInnen spezifisch weiterzubilden und kreislaufwirtschaftlich orientierte Trainings anzubieten. Diese könnten allerdings - neben der Einstellung spezifischer Fachkräfte - für eine Integration kreislaufwirtschaftlicher Aspekte in verschiedene Abteilungen führen und so Silo-Denken überwinden.

Als vierter Bereich der strategischen Orientierung wurden betriebliche Voraussetzungen in den Blickpunkt genommen. Hier wird sichtbar, dass Unternehmen generell von sich behaupten, gut auf kreislaufwirtschaftliche Anpassungen vorbereitet $\mathrm{zu}$ sein. Dies trifft vor allen Dingen auf vorhandene physische Ressourcen wie Betriebsstätten, Maschinerie und Anlagen, oder auch Eigentum zu. Hier denken $43 \%$, dass die Voraussetzungen unternehmensweit gegeben sind oder vorbereitet werden, weitere $20 \%$ sehen dies in Einzelfällen gegeben. Hinsichtlich der Existenz von Prozessen zur Umsetzungsunterstützung geben $60 \%$ an, dass dies zumindest in Einzelfällen der Fall sei - beide Aspekte müssen nicht nur kreislaufwirtschaftsspezifisch gelesen werden, sondern können auch mit dem Aufbau des Unternehmens oder dem Geschäftsbetrieb zusammenhängen. Etwas weniger überzeugt von ihrer Bereitschaft zur Umsetzung von
Kreislaufwirtschaft sind Unternehmen, wenn es um digitale Technologien und IT-Systeme geht. Trotzdem gaben auch hier immerhin noch $53 \%$ der Befragten an, dass diese zumindest in einzelnen Bereichen bereits ausreichend sei $37 \%$ gaben an, dass auch unternehmensweit bereits die notwendigen Voraussetzungen vorbereitet würden oder bereits umgesetzt sind).

Zusammenfassend lässt sich sagen, dass das Thema Kreislaufwirtschaft teilweise auch auf strategischer Ebene in österreichischen Unternehmen angekommen ist. Was jedoch konkret unternommen werden soll oder welche Aspekte von Kreislaufwirtschaft wie und in welchem zeitlichen Rahmen umgesetzt werden sollen, wird bislang nicht transparent gemacht und könnte daher auch ein Zeichen sein, dass den Unternehmen noch nicht klar ist, was sie in Richtung einer Kreislaufwirtschaft unternehmen könnten. Auch die Allokation von Humanressourcen in Form von Weiterbildung von MitarbeiterInnen scheint in diesem Zusammen- 


\section{Effekt von Nachhaltigkeits- und Kreislaufwirtschaftsaktivitäten auf die Nachhaltigkeitsleistung}

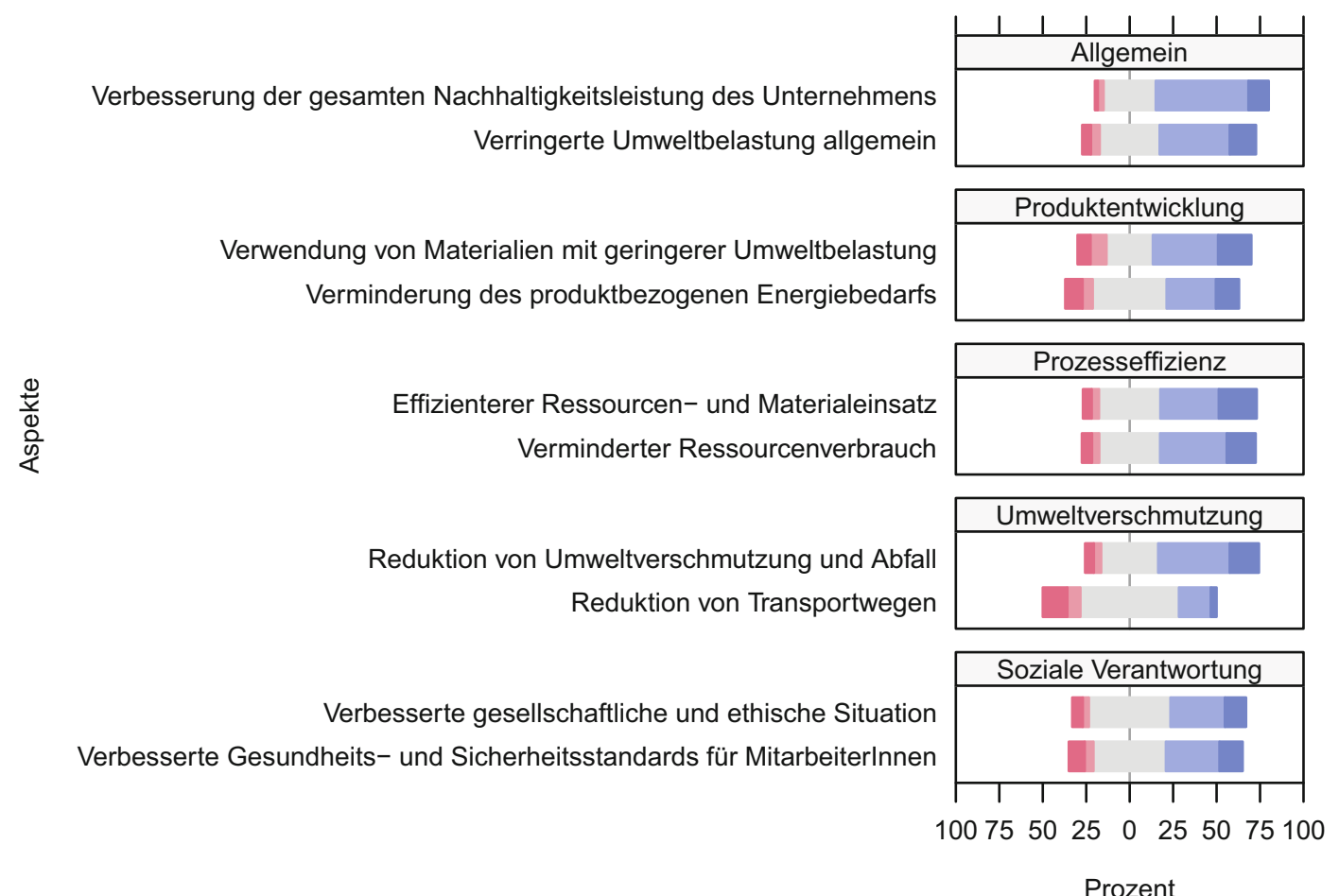

Abb. 7 Einfluss von Nachhaltigkeits- und Kreislaufwirtschaftsaktivitäten auf die Nachhaltigkeitsleistung der befragten Unternehmen ( $n=120$ GeschäftsführerInnen); Balkenfarben von links nach rechts: dunkelrot-stark verschlechtert; rot-verschlechtert; grau neutral; blau -verbessert; dunkelblau -stark verbessert

hang noch nicht in gleicher Weise von den Unternehmen aufgenommen.

4.4 Effekte von Nachhaltigkeits- und Kreislaufwirtschaftsaktivitäten auf die Nachhaltigkeitsleistung und den Unternehmenserfolg

Wie in Kap. 3 beschrieben, wurden zusätzlich zu den 100 Führungskräften mit Verantwortlichkeiten zu Nachhaltigkeitsthemen auch 120 GeschäftsführerInnen $\mathrm{zu}$ den Effekten der in den Abschn. 4.1 und 4.2 beschriebenen Nachhaltigkeits- und Kreislaufwirtschaftsaktivitäten auf den Unternehmenserfolg befragt. Dabei wurde zwischen Nachhaltigkeitsleistung und ökonomischen Unternehmenserfolg unterschieden und konkret nach dem Einfluss der verschiedenen Aktivitäten der letzten drei Jahre auf diese beiden Ergebnisgrößen gefragt.

\subsubsection{Effekte auf die Nachhaltigkeitsleistung}

Wie in Abb. 7 ersichtlich, können die Effekte auf die Nachhaltigkeitsleistung in (i) Allgemeine, (ii) Produktentwicklung, (iii) Prozesseffizienz, (iv) Umweltverschmutzung und (v) soziale Verantwortung unterteilt werden. Generell zeigt sich, dass knapp zwei Drittel (65\%) der GeschäftsführerInnen angeben, dass sich die Nachhaltigkeits- und Kreislaufwirtschaftsaktivitäten ihres Unternehmens der letzten drei Jahre positiv (53\%) oder sehr positiv (12\%) auf die Nachhaltigkeitsleistung des Unternehmens ausgewirkt haben. Etwas weniger (58\%) gaben zudem an, dass sich durch die Aktivitäten auch die generellen Umweltauswirkungen ihres Unternehmens verringert haben. Ein ähnliches, mehrheitlich positives Bild zeigt sich auch bei drei der vier spezifischeren Kategorien. Lediglich in der Kategorie der sozialen Verantwortung sahen weniger als die Hälfte der GeschäftsführerInnen eine Verbesserung der Gesundheitsund Sicherheitsstandards für Mitarbei-
terInnen (44\%) oder der gesellschaftlichen und ethischen Situation $(43 \%)$.

Wie die Häufigkeit der Antworten auf die Antwortmöglichkeit „Neutral“ zeigen, haben die Nachhaltigkeits- und Kreislaufwirtschaftsaktivitäten in der Mehrheit der Fälle keinen Effekt auf die Verringerung von Transportdistanzen (57\% Neutral) und die neutrale Antwortmöglichkeit überwiegt mögliche negative und positive Effekte auch hinsichtlich der Verbesserung der gesellschaftlichen und ethischen Situation (47\%) und der Verminderung des produktbezogenen Energiebedarfs (42\%). Negative Effekte auf die Nachhaltigkeitsleistung sind generell seltener $\mathrm{zu}$ beobachten (5-22\% kumulierte Häufigkeit der beiden negativen Antwortkategorien) und betreffen vor allem die Transportdistanzen (14\% stark negativer Effekt) sowie den Energiebedarf der Produkte $(10 \%$ stark negativer Effekt) und die Gesundheits- und Sicherheitsstandards der MitarbeiterInnen (9\% stark negativer Effekt). Zusammengefasst zeigt sich aus Sicht der befragten GeschäftsführerInnen eine 


\section{Effekt von Nachhaltigkeits- und Kreislaufwirtschaftsaktivitäten auf den Unternehmenserfolg}

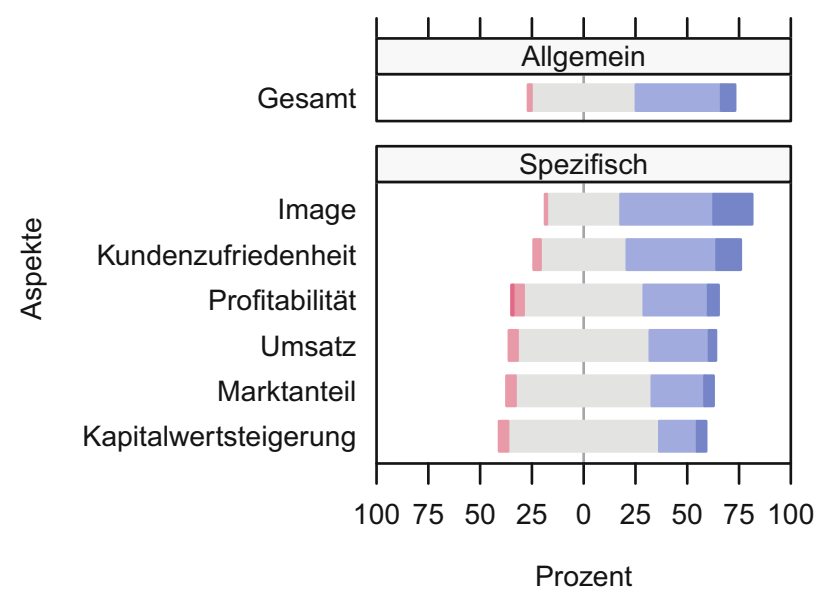

Abb. 8 Effekt von Nachhaltigkeits-und Kreislaufwirtschaftsaktivitäten auf den finanziellen Erfolg der befragten Unternehmen ( $n=120$ GeschäftsführerInnen); Balkenfarben von links nach rechts: dunkelrot - stark verschlechtert; rot-verschlechtert; grau - neutral; blau-verbessert; dunkelblau -stark verbessert

durch Nachhaltigkeits- und Kreislaufwirtschaftsaktivitäten der letzten drei Jahre induzierte generelle Verbesserung der Nachhaltigkeitsleistung der Unternehmen. Hinsichtlich Verbesserungen in konkreten Bereichen liegt diese Mehrheit aber in sechs von zehn Fällen nur knapp über der Hälfte (55-58\%). In den restlichen vier Fällen, zu denen die beiden sozialen Aspekte sowie die Transportdistanzen und der produktbezogene Energiebedarf zählen, zeigen sich mehrheitlich keine Effekte.

\subsubsection{Effekte auf den} Unternehmenserfolg

Abb. 8 stellt die Ergebnisse der Frage hinsichtlich des Effekts der Nachhaltigkeits- und Kreislaufwirtschaftsaktivitäten auf den ökonomischen Unternehmenserfolg dar. Die Antworten gliedern sich dabei in einen allgemeinen Effekt und sechs spezifische Effekte. Allgemein sehen weniger als die Hälfte der befragten GeschäftsführerÍnnen $(48 \%)$ einen positiven $(41 \%)$ oder stark positiven Effekt (7\%) der Nachhaltigkeitsund Kreislaufwirtschaftsaktivitäten auf den Unternehmenserfolg.

Vergleicht man die Ergebnisse in Abb. 7 und 8 ergibt sich, dass die GeschäftsführerInnen etwa $10 \%$ seltener einen positiven Effekt auf den Unternehmenserfolg als auf die Nach-
4.5 Einschätzung des österreichischen Markts im Kontext einer Kreislaufwirtschaft

Abschließend wurden die GeschäftsführerInnen um Einschätzungen ihres Marktumfeldes hinsichtlich der Umsetzung einer Kreislaufwirtschaft in Österreich ersucht. Wie in Abb. 9 dargestellt, stimmt eine überwiegende Mehrheit der Aussage zu, dass technischen Entwicklungen ein großes Potenzial für die Umsetzung einer Kreislaufwirtschaft in Österreich aufweisen. Auf die Frage, ob diese Technologien auch schon zur Realisierung von zirkulären Produktideen beigetragen haben, antworteten jedoch nur etwas mehr als ein Drittel (36\%) der GeschäftsführerInnen zustimmend und etwas weniger als ein Drittel stimmten dieser Aussage nicht oder überhaupt nicht zu (32\%). Ein großteils positives Bild zeigt sich bezüglich der Marktentwicklungen. Hier geben jeweils etwa ein Drittel der Befragten an, dass KundInnen generell empfänglich $(66 \%)$ für zirkuläre Produktideen wären bzw. diese in den jeweiligen Industriesektoren auch erwarten würden (59\%). Bezüglich der Wettbewerbsintensität hin zu einer Kreislaufwirtschaft zeigt sich, dass vor allem von Seiten der öffentlichen Hand eine Erwartungshaltung zur Umsetzung von Kreislaufwirtschaftsinitiativen wahrgenommen wird (57\%). Während die Antworten auf die Frage zum direkten Wettbewerb generell gemischter ausfallen, berichtet immerhin schon etwa ein Drittel der GeschäftsführerInnen von einem intensiven Wettbewerb zu zirkulären und nachhaltigen Ideen (34\%) und ebenfalls ein Drittel (32\%) gibt an, dass die MitbewerberInnen bereits zirkuläre Produkte und Services eingeführt haben.

\section{Diskussion}

Die Implementierung von Nachhaltigkeitsstrategien ist in den meisten Unternehmen bereits im Gange; entweder wurden diese Nachhaltigkeitsstrategien schon unternehmensweit umgesetzt, eine unternehmensweite Umsetzung befindet sich im Gange oder erste Umsetzungen in Pilotprojekten wurden gestartet (Abb. 3). Dies lässt darauf schließen, dass Nachhaltigkeitsthemen bei österreichischen Produktionsunternehmen auch längerfristig noch auf der Agenda stehen und an Bedeutung zunehmen werden. Allerdings werden Zertifizierungen, bzw. damit 


\section{Markteinschätzung zur Kreislaufwirtschaft in Österreich}

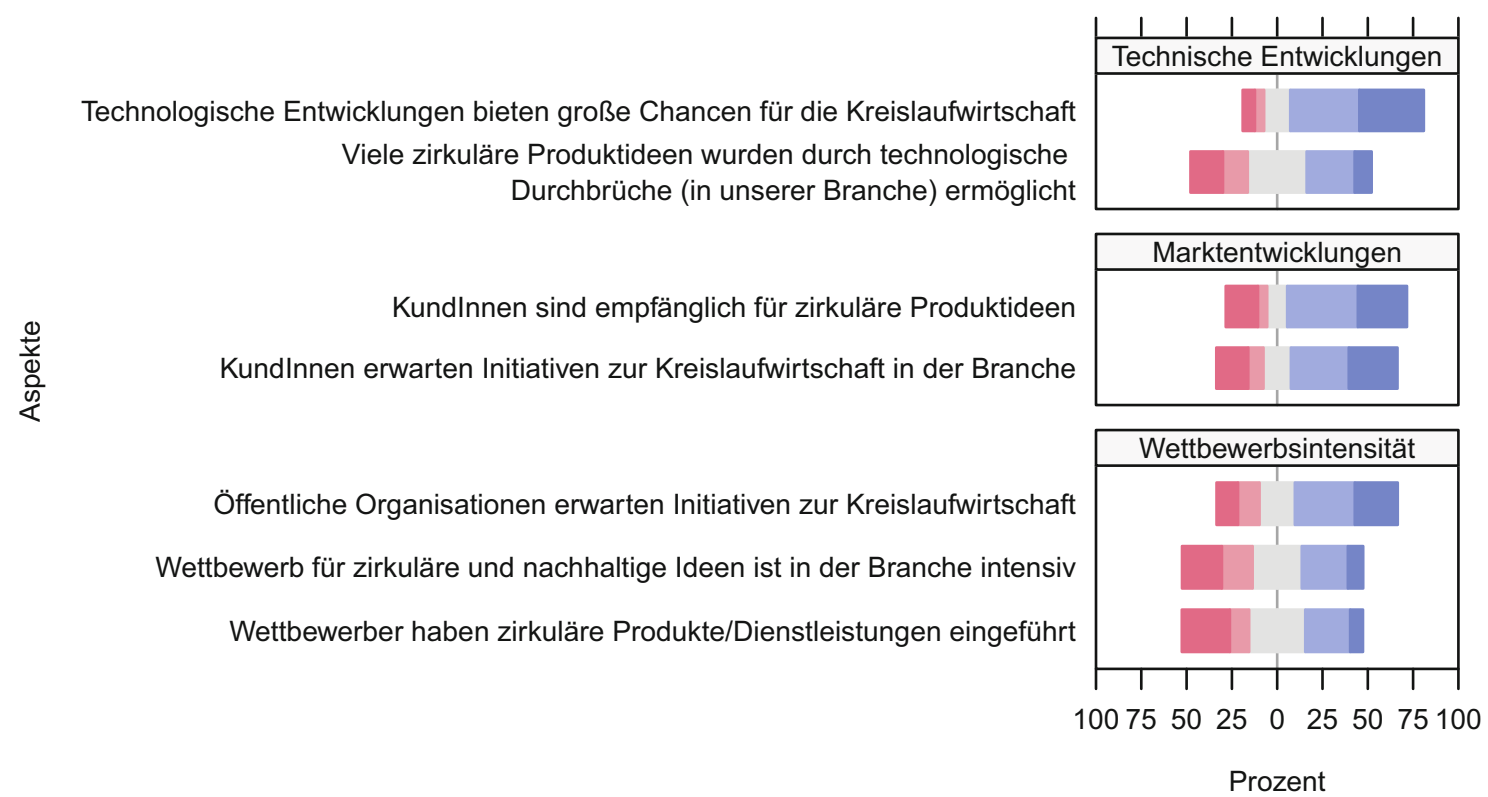

Abb. 9 MarkteinschätzungzurKreislaufwirtschaftinÖsterreich ( $n=120$ Geschäftsführerlnnen); Balkenfarbenvon linksnach rechts: dunkelrot-stimme überhaupt nicht zu; rot-stimme nicht zu; grau - neutral; blau -stimme zu; dunkelblau - stimme voll und ganz zu

einhergehende kontinuierliche Verbesserungsmaßnahmen wie ISO 14001, OHSAS 18001, ISO 50001 und EMAS erst von wenigen der befragten Unternehmen genutzt.

Bei der Implementierung von konkreten Nachhaltigkeits- und Kreislaufwirtschaftspraktiken (Abb. 4) wird deutlich, dass vor allem die Steigerung der Material- und Energieeffizienz von den Unternehmen angestrebt wird. Ebenso wichtig sind Compliance Themen für die Unternehmen, wie z.B. die Vermeidung von toxischen Stoffen in Produkten. Der am häufigsten umgesetzte Aspekt in der Kategorie „Design“ ist die Berücksichtigung von ökologischen Aspekten im Produktdesign. Eine klare Abgrenzung zu anderen Design-Aspekten ist hier jedoch schwierig. Es wurde nicht abgefragt, wie diese Aspekte von den Unternehmen verstanden und umgesetzt werden. Die Produkt Compliance kann als Grundlage für weitere Nachhaltigkeitsbestrebungen auf Produktebene angesehen werden. Darauf aufbauend können Überlegungen folgen, wie z.B. design for recycling, das über die gesetzlichen Vorschriften hinausgeht, vorangetrieben werden kann. Recyclingfreundliches Design wird von vielen produzierenden Unternehmen bereits umgesetzt. Etwas seltener werden in den Firmen Sekundärrohstoffe bzw. recycelte Materialien und „postconsumer" Produkte für die eigene Produktion verwendet. Durch die Verknüpfung dieser beiden Themen und einem entschiedenen Einsatz von Sekundärrohstoffen in der eigenen Produktion könnten sich weitere Synergien ergeben.

Obwohl die Bewertung der Kreislauffähigkeit von Produkten eine fast ebenso wichtige Rolle spielt wie die Bewertung der ökologischen Produktaspekte, wird durch die Ergebnisse zur Verfügbarkeit von Informationen zum Recycling (Abb. 5) verdeutlicht, dass derzeit nur wenig Daten aus der Endof-Life Phase in produzierenden Unternehmen vorhanden sind, um eben eine solche Bewertung durchführen zu können. Sollen jedoch messbare kreislaufwirtschaftliche Ziele definiert werden, wird gleichzeitig auch die Notwendigkeit einer höheren Datenverfügbarkeit gegeben sein. Da bisher noch Informationslücken vorhanden sind, werden auch noch wenig konkrete Ziele von den Unternehmen veröffentlicht. Mehr Transparenz (beispielsweise bezogen auf die Veröffentlichung kreislaufwirtschaftlicher Zielsetzungen in Unternehmen) wird entsprechend nur einhergehend mit einer verbesserten Datenverfügbarkeit, ergo mit einem verbesserten und nachvollziehbaren
Datenaustausch entlang der Lieferkette, möglich sein. So kann dem Ansatz generischer Durchschnittswerte, wie derzeit häufig in Ökobilanzen erkennbar, bereits ex ante entgegengewirkt werden.

Da mehr als die Hälfte der Unternehmen das eigene Innovationspotenzial nicht für ausreichend halten (Abb. 6), um zirkuläre Geschäftsmodelle, Produkte, oder Services zu implementieren, kann angenommen werden, dass Kooperationen (Barreiro-Gen und Lozano 2020) und „open-innovation“ Projekte (Rauter et al. 2019) in Zukunft an Bedeutung gewinnen werden. Trotz des nicht ausreichenden Innovationspotenzials geben die Unternehmen an, betriebliche Voraussetzungen für kreislaufwirtschaftliche Anpassungen zu erfüllen (z.B. von Betriebsstätten, Maschinerie und Anlagen, oder auch Eigentum) sowie eine Nachhaltigkeitsstrategie $\mathrm{zu}$ verfolgen. Wenn es um die zur Verfügung stehenden digitalen Technologien und IT-Systeme zur Umsetzung einer Kreislaufwirtschaft geht, sind etwa die Hälfte der Unternehmen der Meinung, bereits über die geeigneten Mittel zu verfügen. Eine überwiegende Mehrheit der GeschäftsführerInnen sieht zudem in technischen Entwicklungen ein großes Potenzial für die 
Umsetzung einer Kreislaufwirtschaft in Österreich (Abb. 9).

Angesichts der immer wichtiger werdenden Rolle von digitalen Technologien in der Umsetzung einer CE, die zumindest in der Literatur schon deutlich wird (Rusch et al. 2021; Schöggl et al. 2020a), sollte in digitale Technologien investiert werden bzw. die Entwicklung in den unterschiedlichen Branchen genau beobachtet werden.

\section{Schlussfolgerungen}

Diese Studie hatte eine sektorübergreifende Erhebung des Umsetzungsgrades einer nachhaltigen Kreislaufwirtschaft in österreichischen Produktionsunternehmen zum Ziel. Zu diesem Zweck wurden 100 Führungskräfte im Nachhaltigkeitsbereich sowie 120 GeschäftsführerInnen mittels strukturierten Telefoninterviews befragt. Die Studie zeigt, dass die befragten Unternehmen bei der Implementierung von Nachhaltigkeits- und Kreislaufwirtschaftspraktiken einen Fokus auf die Themen Compliance und Effizienz legen. Obwohl ein großer Teil der befragten Unternehmen bereits Nachhaltigkeitsstrategien definiert haben, sind radikale, kreislaufwirtschafts-orientierte Änderungen der Geschäftsprozesse und -modelle bisher eher selten. Diese radikalen Änderungen sind unter anderem auch mit dem Innovationspotential der Unternehmen verknüpft, das in einigen Firmen noch nicht vorhanden ist. Aus Sicht der befragten GeschäftsführerInnen wirken sich Nachhaltigkeits- und Kreislaufwirtschaftsaktivitäten jedoch bereits großteils positiv auf die Nachhaltigkeitsleistung ihrer Unternehmen und teilweise positiv auf den ökonomischen Unternehmenserfolg aus. Die öffentliche Hand wird, neben einer zunehmenden Empfänglichkeit von KundInnen für zirkuläre Produkte, am häufigsten als Treiber von kreislaufwirtschaftlichen Entwicklungen am österreichischen Markt gesehen. Einigkeit herrscht auch hinsichtlich der zentralen Rolle digitaler Technologien, welche ein großes, aber weitgehend noch nicht realisiertes Potenzial darstellen. Recycling als eine zentrale Kreislaufwirtschaftsstrategie wird von den befragten Unternehmen hauptsächlich unternehmensintern verfolgt. Die Wiederverwendung oder das Recycling von „post-consumer“ Produkten ist weniger weit verbreitet. Mit Hinsicht darauf zeigt diese Studie auch, dass Unternehmen noch kaum Informationen über die Verwertungswege ihrer Produkte im End-of-Life haben. Die Schließung dieser Informationslücke und eine digitale Vernetzung von verschiedenen Akteuren entlang multipler Produkt- und Materiallebenszyklen kann neben technologischen Entwicklungen im Bereich des Recyclings als ein zentrales Entwicklungsziel in der Transition zu einer Kreislaufwirtschaft angesehen werden.

Die Ergebnisse der Studie können als Grundlage für weitere Forschungsvorhaben dienen, in welchen etwa die Treiber und Potenziale der Umsetzung einer nachhaltigen Kreislaufwirtschaft in österreichischen Unternehmen weiter detailliert werden. Eine Limitation dieser Studie liegt in der rein deskriptiven Analyse von sektorübergreifenden Daten. Aufgrund dieser bleiben mögliche Unterschiede zwischen den Sektoren hinsichtlich der Strategien und der Auffassung einer nachhaltigen Kreislaufwirtschaft sowie der Einfluss der Unternehmensgröße und Wirkgefüge zwischen verschiedenen Faktoren verborgen. Weiterführende Forschung wird sich jedoch einer tiefergehenden inferenzstatistischen Analyse der Daten widmen.

Funding The financial support of the Austrian Federal Ministry for Digital and Economic Affairs, the National Foundation for Research, Technology and Development, and the Christian Doppler Research Association, is gratefully acknowledged. Open access funding was provided by University of Graz.

Open Access Dieser Artikel wird unter der Creative Commons Namensnennung 4.0 International Lizenz veröffentlicht, welche die Nutzung, Vervielfältigung, Bearbeitung, Verbreitung und Wiedergabe in jeglichem Medium und Format erlaubt, sofern Sie den/die ursprünglichen Autor(en) und die Quelle ordnungsgemäß nennen, einen Link zur Creative Commons Lizenz beifügen und angeben, ob Änderungen vorgenommen wurden.

Die in diesem Artikel enthaltenen Bilder und sonstiges Drittmaterial unterliegen ebenfalls der genannten Creative Commons Lizenz, sofern sich aus der Abbildungslegende nichts anderes ergibt. Sofern das betreffende Material nicht unter der genannten Creative Commons Lizenz steht und die betreffende Handlung nicht nach gesetzlichen Vorschriften erlaubt ist, ist für die oben aufgeführten Weiterverwendungen des Materials die Einwilligung des jeweiligen Rechteinhabers einzuholen.

Weitere Details zur Lizenz entnehmen Sie bitte der Lizenzinformation auf http://creativecommons.org/licenses/ by/4.0/deed.de.

\section{Literatur}

Barreiro-Gen, M., \& Lozano, R. (2020): How circular is the circular economy? Analysing the implementation of circular economy in organisations. Business Strategy and the Environment, 29(8), 3484-3494. https://doi.org/10.1002/bse. 2590

Bocken, N. M. P., de Pauw, I., Bakker, C., \& van der Grinten, B. (2016): Product design and business model strategies for a circular economy Journal of Industrial and Production Enginee ring, 33(5), 308-320. https://doi.org/10.1080/ 21681015.2016.1172124

Bocken, N. M. P., Ritala, P., \& Huotari, P. (2017) The Circular Economy: Exploring the Introduction of the Concept Among S\&P 500 Firms. Journal of Industrial Ecology, 21(3), 487-490. https://doi. org/10.1111/jiec.12605
Bundesgesetz über eine nachhaltige Abfallwirtschaft (2021): Austria.

Bundesministerium für Nachhaltigkeit und Tourismus. (2019): Masterplan Umwelttechnologie. Wien.

Cecchin, A., Salomone, R., Deutz, P., Raggi, A., \& Cutaia, L. (2021): What Is in a Name? The Rising Star of the Circular Economy as a ResourceRelated Concept for Sustainable Development. Circular Economy and Sustainability, 1(1), 83-97. https://doi.org/10.1007/s43615-021-00021-4

Circle Economy, \& ARA. (2019): The Circularity Gap Report-Austria. Vienna.

Corvellec, H., Stowell, A.F., \& Johansson, N. (2021): Critiques of the circular economy. Journal of Industrial Ecology, jiec.13187. https://doi. org/10.1111/jiec.13187
Deponieverordnung (2021).

European Commission. (2015): Closing the loop-An EU action plan for the Circular Economy. Brussels. Retrieved from http://eur-lex. europa.eu/legal-content/EN/TXT/?uri=CELEX: 52015DC0614. Zugegriffen: 13. Okt. 2021

European Commission. 2019: The European Green Deal (2019). Brussels.

European Commission. (2020): A new Circular Economy Action Plan-For a cleaner and more competitive Europe, Pub. L. No. COM(2020) 98 final (2020). Brussels.

Figge, F., \& Thorpe, A. S. (2019): The symbiotic rebound effect in the circular economy. Ecological Economics, 163(April), 61-69. https://doi. org/10.1016/j.ecolecon.2019.04.028 
Geissdoerfer, M., Morioka, S. N., de Carvalho, M. M., \& Evans, S. (2018): Business models and supply chains for the circular economy. Jour nal of Cleaner Production, 190(April), 712-721. https://doi.org/10.1016/j.jclepro.2018.04.159

Geissdoerfer, M., Savaget, P., Bocken, N. M. P. \& Hultink, E. J. (2017): The Circular Economy-a new sustainability paradigm? Journal of Cleaner Production, 143(under review), 757-768. https://doi.org/10.1016/j.jclepro.2016.12.048

Govindan, K., \& Hasanagic, M. (2018): A systematic review on drivers, barriers, and practices towards circular economy: a supply chain perspective. International Journal of Production Research, 56(1-2), 278-311. https://doi.org/10. 1080/00207543.2017.1402141

Heshmati, A. (2015): A Review of the Circular Economy and its Implementation. Seoul.

Holzer, D., Rauter, R., Fleiß, E., \& Stern, T. (2021): Mind the gap: Towards a systematic circular economy encouragement of small and medium-sized companies. Journal of Cleaner Production, 298, 126696. https://doi.org/10. 1016/j.jclepro.2021.126696

International Resource Panel. (2019): Global Resources Outlook 2019: Natural Resources for the Future We Want. (B. Oberle, S. Bringezu, S. Hatfield-Dodds, S. Hellweg, H. Schandl, J. Clement, ... B. Zhu, Eds.). Nairobi: International Resource Panel; United Nations Environment Programme. https://doi.org/10.18356/ 689ala17-en

Khan, O., Daddi, T., \& Iraldo, F. (2020): The role of dynamic capabilities in circular economy implementation and performance of companies Corporate Social Responsibility and Environmental Management, (July), 3018-3033. https:// doi.org/10.1002/csr.2020

Ellen MacArthur Foundation. (2015): Growth within: a circular economy vision for a competitive europe. Ellen MacArthur Foundation, SUN McKinsey Center for Business and Environment Ellen MacArthur Foundation. (2020): Circulytics: Overview.
Mura, M., Longo, M., \& Zanni, S. (2020): Circular economy in Italian SMEs: A multi-method study. Journal of Cleaner Production, 245 , 118821. https://doi.org/10.1016/j.jclepro.2019. 118821

Ökodesign-Verordnung (2011).

Ormazabal, M., Prieto-Sandoval, V., Puga-Leal, R., \& Jaca, C. (2018): Circular Economy in Spanish SMEs: Challenges and opportunities. Journal of Cleaner Production, 185, 157-167. https:// doi.org/10.1016/j.jclepro.2018.03.031

Rauter, R., Globocnik, D., Perl-Vorbach, E., \& Baumgartner, R. J. (2019): Open innovation and its effects on economic and sustainability innovation performance. Journal of Innovation and Knowledge, 4(4), 226-233. https://doi.org/ $10.1016 / j . j i k .2018 .03 .004$

Recycling-Baustoffverordnung (2015)

Reike, D., Vermeulen, W. J. V., \& Witjes, S. (2018): The circular economy: New or Refurbished as CE 3.0?-Exploring Controversies in the Conceptualization of the Circular Economy through a Focus on History and Resource Value Retention Options. Resources, Conservation and Recycling, 135(5), 246-264. https://doi.org/10. 1016/j.resconrec.2017.08.027

Rusch, M., Schöggl, J.-P., \& Baumgartner, R. J. (2021): Application of digital technologies for sustainable product management in a circular economy-a review. Business Strategy and the Environment. Article in press. https://doi.org/ 10.31235/osf.io/twgks

Santa-Maria, T., Vermeulen, W. J. V., \& Baumgartner, R. J. (2021): Framing and assessing the emergent field of business model innovation for the circular economy: A combined literature review and multiple case study approach. Sustain able Production and Consumption, 26, 872-891. https://doi.org/10.1016/j.spc.2020.12.037

Schöggl, J.-P., Baumgartner, R. J., \& Hofer, D. (2017): Improving sustainability performance in early phases of product design: A checklist for sustainable product development tested in the automotive industry. Journal of Cleaner Produc- tion, 140, 1602-1617. https://doi.org/10.1016/j. jclepro.2016.09.195

Schöggl, J.-P., Rusch, M., Schiffleitner, A., \& Baumgartner, R. J. (2020a): What to expect from data-driven sustainable product manage-ment? Insights from industry cases and PLM solution providers. In Fraunhofer Institute for Reliability and Microintegration IZM (Ed.), Electronics Goes Green 2020+ (pp. 637-643). Berlin: Fraunhofer-Verlag.

Schöggl, J.-P., Stumpf, L., \& Baumgartner, R. J. (2020b): The narrative of sustainability and circular economy-A longitudinal review of two decades of research. Resources, Conservation and Recycling, 163, 105073. https://doi.org/10.1016/ j.resconrec.2020.105073

Stumpf, L., Schöggl, J.-P., \& Baumgartner, R. J. (2021): Climbing up the circularity ladder?-A mixed-methods analysis of circular economy in business practice. Journal of Cleaner Production 316, 128158. https://doi.org/10.1016/j.jclepro. 2021.128158

Tschulik, A. (2021): Österreichische Strategie für Kreislaufwirtschaft. Bundesministerium für $\mathrm{Kli}$ maschutz, Umwelt, Energie, Mobilität, Innovation und Technologie.

Verpackungsverordnung (2014).

Walker, A. M., Opferkuch, K., Roos Lindgreen, E., Simboli, A., Vermeulen, W. J. V., \& Raggi, A. (2021): Assessing the social sustainability of circular economy practices: Industry perspectives from Italy and the Netherlands. Sustainable Production and Consumption, 27, 831-844. https:// doi.org/10.1016/j.spc.2021.01.030

Zink, T., \& Geyer, R. (2017): Circular Economy Rebound. Journal of Industrial Ecology, 21(3), 593-602. https://doi.org/10.1111/jiec.12545

Hinweis des Verlags Der Verlag bleibt in Hinblick auf geografische Zuordnungen und Gebietsbezeichnungen in veröffentlichten Karten und Institutsadressen neutral. 